\title{
Control de la distancia inter electrodos en un proceso de micro maquinado electroquímico por pulsos (PECMM)
}

\author{
Iván Gallegos Miranda, José Arturo Pérez Martínez, Everardo Efrén Granda Gutiérrez, \\ Juan Fernando García Mejía \\ Universidad Autónoma del Estado de México, \\ Toluca, Estado de México, México \\ igallegosmiranda@outlook.es, japerezm@uaemex.mx, \\ eegrandag@uaemex.mx, fgarciam@uaemex.mx
}

Resumen. El micro maquinado electroquímico por pulsos (PECMM), es un proceso de manufactura no convencional, en donde la herramienta de trabajo no tiene desgaste, por lo que ofrece una gran ventaja para fabricar piezas de un tamaño reducido, en comparación con los procesos convencionales. El mantener las condiciones óptimas del proceso PECMM garantiza mayor eficiencia y rendimiento de maquinado, donde una de las condiciones críticas del proceso es mantener constante la distancia entre el electrodo y la pieza de trabajo (distancia entre electrodos) para garantizar la remoción homogénea del material. En este documento se presenta el diseño y aplicación de un controlador por lógica difusa (FLC) tipo SISO (del inglés, una entrada una salida) con tres conjuntos difusos de entrada y tres de salida, cuyo objetivo es asegurar que la distancia entre electrodos se mantenga en el orden de $240 \mu \mathrm{m}$. La implementación de un controlador de este tipo demuestra ser una alternativa eficaz para esta tarea, principalmente debido a que la distancia entre electrodos no es una variable observable directamente, sino que se debe estimar a partir de otras variables del proceso. Lo anterior se realiza mediante la medición de la corriente de maquinado. Los resultados muestran que el controlador permite ajustar progresivamente la distancia del electrodo, por lo que se obtiene un canal de profundidad homogénea, inclusive si la superficie presenta irregularidades a lo largo de la trayectoria de desplazamiento del electrodo, con una precisión cercana a $10 \mu \mathrm{m}$.

Palabras clave: control por lógica difusa, micro-maquinado electroquímico por pulsos, procesos de manufactura.

\section{Gap Control of a Process of Micro Electrochemical Machining by Pulses (PECMM)}

\begin{abstract}
Pulsed electrochemical micro machining (PECMM) is a nonconventional manufacturing process where the tool does not present wear, offering a considerable advantage over conventional processes on the machining of small pieces. In order to guarantee the proper efficiency and performance, the process working parameters must be kept in optimal intervals. One of the critical constraints is to keep constant the distance between the workpiece and the tool
\end{abstract}


electrode (gap) to ensure a homogeneous material removal. With the aim to keep the gap in the order of $240 \mu \mathrm{m}$, in this work is proposed the design and application of a SISO (single-input single output) Fuzzy Logic Controller (FLC) with three fuzzy sets for both: input and output. The implementation of such a controller demonstrates to be a good alternative performing this task, mainly because the gap is not an observable variable, but it could be estimated from other process variables. Thus, the machining current has been used in this investigation. Results show that the FLC allows to continuously adjust the inter electrode distance, machining a channel with homogeneous depth, even though the surface presents irregularities along the electrode trajectory, reaching a precision close to $10 \mu \mathrm{m}$.

Keywords: fuzzy logic control, pulsed electrochemical micro-machining (PECMM), manufacturing process.

\section{Introducción}

La creciente demanda en la fabricación de componentes de tamaño reducido en áreas como robótica, biología, micro electrónica, material biomédico, entre otros; da auge a la investigación en el desarrollo de procesos de micro manufactura [1]. Como aplicaciones de este proceso se puede mencionar la fabricación de micro boquillas, micro engranes, instrumentos quirúrgicos (bisturíes), micro tubos, micro inyectores y micro bujes [2].

El proceso de micro manufactura, puede realizarse mediante métodos convencionales (se genera fricción entre la herramienta y la pieza de trabajo) y no convencionales (se realiza la remoción de material sin contacto ente la herramienta y la pieza de trabajo). El proceso de manufactura convencional, se genera mediante fricción, generando micro fisuras y residuos metálicos, esto es una desventaja con respecto a los métodos no convencionales, que se caracterizan por la ausencia de fricción y generación de residuos [3].

El proceso de micro maquinado electroquímico por pulsos (PECMM por sus siglas en ingles), es un proceso de manufactura no convencional donde la herramienta de trabajo no tiene contacto con la pieza de trabajo, por lo que siempre existe una separación entre ambas a esto se le denomina distancia inter electrodos ( $g$ ap $)$. PECMM es una técnica que aprovecha el efecto electrolítico y la corriente eléctrica para provocar la remoción de material por disolución anódica, en componentes conductores de la electricidad, principalmente materiales metálicos [4].

De acuerdo con los trabajos [5-8], el controlar el proceso de maquinado electroquímico tiene una alta complejidad debido a que los resultados obtenidos al término del maquinado, dependen de la configuración de los parámetros de entrada; voltaje de polarización, distancia inter electrodos, frecuencia y ciclo de trabajo, concentración del electrolito y el flujo del electrolito, mientras que los parámetros de salida resultantes son: la taza de remoción de material (MRR, por sus siglas en ingles Material Removal Rate), Diámetro del Sobre corte (DOC, por sus siglas en ingles Diametral Overcut) y profundidad del maquinado. 
Goud en [9], resalta que el proceso de maquinado electroquímico (ECM por sus siglas en inglés) está sujeto a complicaciones, debido a que no existe instrumento para medir la distancia inter electrodos de manera directa, una opción es, estimarla mediante la corriente de maquinado debido a que es proporcional a dicha distancia [10]. Kolhi et al. en [11], resaltan que el controlar el proceso de ECM utilizando técnicas de control clásicas resulta complicado debido a la necesidad de obtener el modelo matemático e inclusive los resultados obtenidos presentaban diferentes características en cada prueba.

Paul y Hiremath en [12], presentan una alternativa para controlar el proceso EDM (Maquinado por Descargas Eléctricas), mediante el uso de una técnica de Inteligencia Artificial, específicamente con Lógica Difusa, donde el controlador es diseñado en base a parámetros proporcionados por un experto, sin requerir del modelo matemático de la planta. La ventaja que se tiene al utilizar un control por lógica difusa es que asemeja las acciones de acuerdo con el proceso cognitivo del ser humano.

Keasberry y otros en [13], reportan el desarrollo de un sistema de control por lógica difusa para mantener contante la remoción de material en un proceso de maquinado electroquímico (ECM), donde afirman que el controlarlo mediante voltaje y presión de inyección del electrolito, resulta insuficiente debido a que el control nunca llego a estabilizarse. Por tal motivo Morteza en [14], presenta un estudio para mantener constante la remoción de material en un proceso ECM basado en simulación, utilizando lógica difusa, donde se resalta que para mantener una remoción de material constante es indispensable conservar la distancia inter electrodos en todo el transcurso del proceso y con ello se garantiza una profundidad homogénea.

En este trabajo se presenta el desarrollo de un control basado en lógica difusa donde se mantiene constante la distancia inter electrodos a través del monitoreo de la corriente de maquinado para el maquinado de micro canales. Los parámetros de trabajo utilizados en este trabajo son: Voltaje de polarización de $5 \mathrm{~V}, 40 \mathrm{kHz}$ como frecuencia de los pulsos, ciclo de trabajo del $50 \%$ y la conductividad del electrolito de $4.7 \Omega * \mathrm{~cm}$, mientras que la distancia inter electrodos inicial del proceso es de $240 \mu \mathrm{m}$. El material utilizado es acero AISI1018, cuya composición química es 0.14 - 0.2 C, 0.6 - 0.9 Mn, Fe balance (\% peso), que es el utilizado para la fabricación de micro boquillas, micro engranes y micro bujes.

\section{Metodología}

El proceso PECMM trata de la remoción de material metálico por disolución anódica evitando la generación de chispas. Para ello se polariza la pieza de trabajo como ánodo y la herramienta de trabajo como cátodo utilizando una fuente pulsada.

En la Fig. 1, se muestran un esquema de los componentes que conforman al sistema de micro maquinado electroquímico por pulsos, que consta de un recipiente en donde se coloca la pieza a maquinar, que estará inmersa dentro de un electrolito formado por una mezcla de $\mathrm{H}_{2} \mathrm{O}$ y NaCl con una concentración de masa del $16.6 \%$ lo cual genera una conductividad de $217.1 \mathrm{mS} / \mathrm{cm}$ o $4.7 \Omega * \mathrm{~cm}$ (medido con un conductímetro HANNA modelo HI5521-02). El electrolito fluye por un tubo de acero inoxidable de 
$700 \mu \mathrm{m}$ de diámetro externo y $490 \mu \mathrm{m}$ de diámetro interno con un flujo aproximado de $1 \mathrm{l} / \mathrm{min}$.

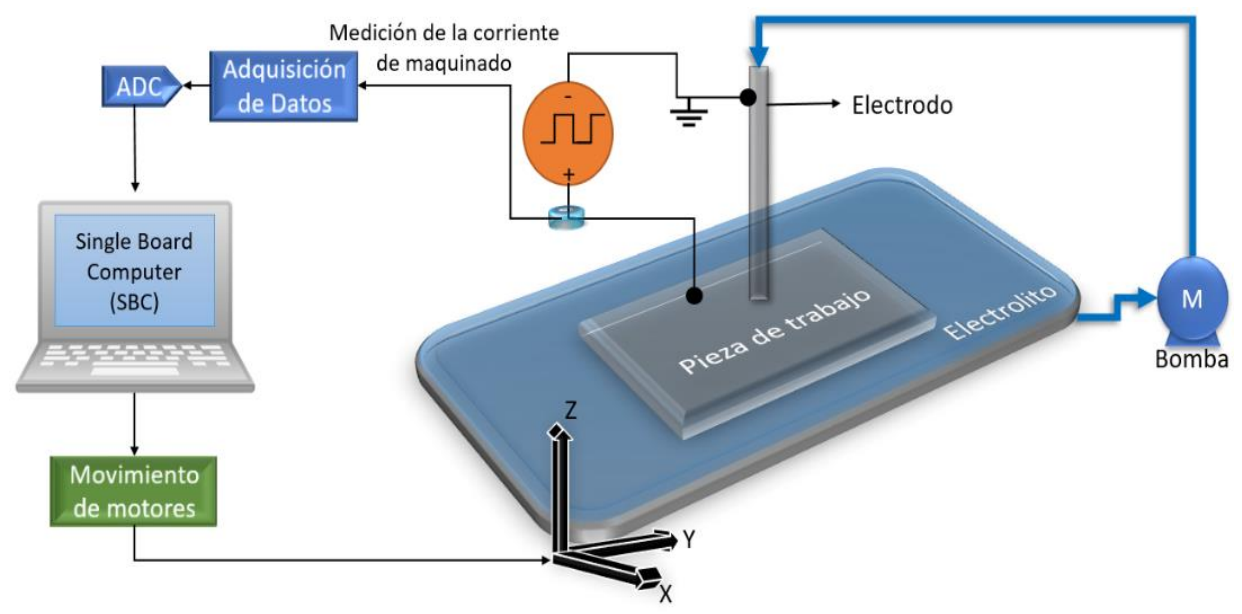

Fig. 1. Diagrama general del Sistema de Micro Maquinado Electroquímico por Pulsos.

La fuente de polarización del proceso es mediante la fuente reportada por [15], la cual se configura para proporcionar un voltaje de $5 \mathrm{~V}$, con ciclo de trabajo de $50 \%$ y la frecuencia de los pulsos es de $40 \mathrm{kHz}$. Mientras que la etapa de adquisición de datos se compone por el integrado AD536 que genera una señal de voltaje proporcional en CD del valor cuadrático medio (RMS) proporcionado por un sensor de efecto Hall (ACS712).

El electrodo es desplazado mediante un sistema mecánico de ejes coordenados (XYZ) acoplados a motores a pasos que son manejados mediante un controlador basado en el circuito integrado L297 y en un arreglo de Mosfets en una topología en puente completo para administrar la energía a los devanados de los motores. La SBC genera la acción de comandar el movimiento de los motores y monitorear el comportamiento de la corriente de maquinado, la cual permite estimar la distancia inter electrodos, dado que es proporcional, de acuerdo con la ecuación 1(Grover [1]):

donde:

$$
g=\frac{A}{I_{R M S} *(R * C)^{\prime}}
$$

$g=$ Distancia inter electrodos.

$A=$ Área de exposición del electrodo.

$I_{R M S}=$ Corriente RMS de Maquinado.

$R=$ Resistividad del electrolito.

$c=\left(\right.$ Densidad $^{2} *$ Velocidad de remoción específica $)=(7.87)^{2 *}\left(3.0^{*} 10^{-2}\right)$, constante que depende del material utilizado.

La acción de control por lógica difusa es realizada por la SBC, que es la encargada de comandar el movimiento en el eje Z; buscando mantener la distancia inter electrodos dentro de un intervalo similar a la distancia inicial. 


\subsection{Diseño del sistema de control difuso}

En la Fig.2 se muestra el diagrama de flujo representativo del proceso de micro maquinado electroquímico por pulsos, donde es necesario establecer los parámetros iniciales del proceso, después inicia el proceso de PECMM y se comienza con el monitoreo de la corriente de maquinado para estimar la distancia inter electrodos y generar una acción de control mediante lógica difusa de acuerdo a la posición en que se encuentre, si durante el proceso se genera una remoción de material, la distancia entre los electrodos aumentará y la corriente de maquinado disminuye. Tal efecto es monitoreado y el sistema detectará el cambio en la corriente, la acción del control es compensar esa disminución en la corriente moviendo el electrodo hacia la pieza de trabajo con lo cual disminuye la distancia entre electrodos hasta que la corriente se incremente y se mantenga en un intervalo determinado por el propio control; de tal manera se pretende que la distancia entre los electrodos se mantenga constante.
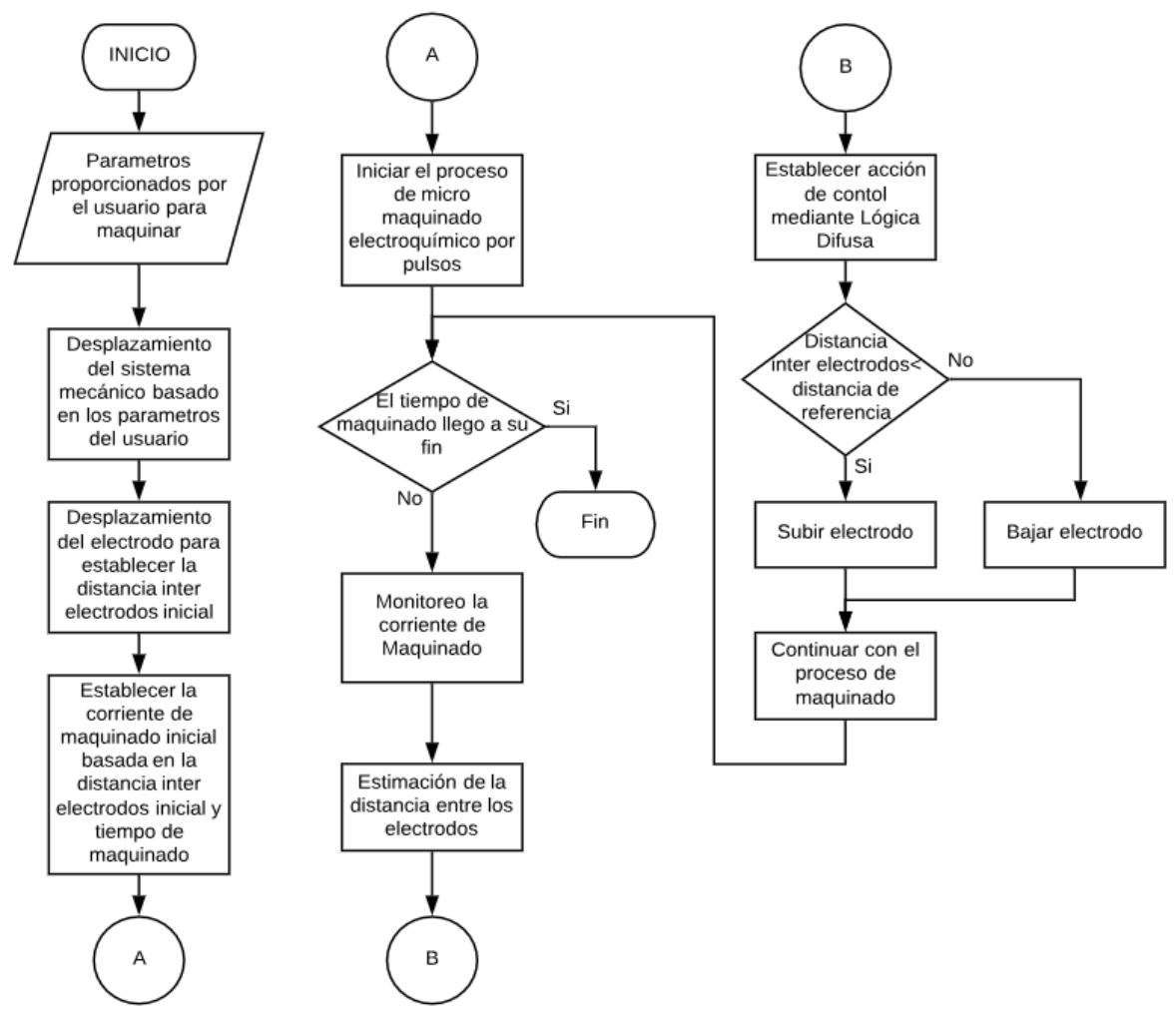

Fig. 2. Diagrama de flujo del proceso PECMM.

Durante el proceso PECMM, es necesario mantener la distancia inter electrodos en un intervalo cercano a los $240 \mu \mathrm{m}$, a fin de obtener remoción de material constante al desplazar el electrodo de forma lineal y generar micro canales cuya profundidad sea la misma, esto es posible controlando la distancia inter electrodos mediante lógica difusa. 
Por tal motivo para el diseño de las reglas de inferencia es necesario contar con la experiencia y el conocimiento de la operación del proceso PECMM, ya que son fuentes de información críticas que se requieren para identificar las condiciones óptimas de operación.

El desarrollo del sistema de control tuvo como base al diseño establecido por Mamdani, y el modelo mediante un sistema SISO (single input- single output), como el mostrado en la Fig. 3. La etapa de entrada contiene 3 funciones de membrecía, mientras que la salida cuenta con tres funciones de membrecía. La entrada al sistema de control es el error de la distancia inter electrodos, que es establecida a través del monitoreo de la corriente de maquinado, mientras que la salida es generar el movimiento del motor a pasos para mantener una la distancia cercana a los $240 \mu \mathrm{m}$. En la Tabla 1 se muestran conjuntos difusos de la entrada o "Error" de la distancia inter electrodos, mientras que en la Tabla 2; se muestran los conjuntos difusos de la distancia requerida para desplazar el electrodo y mantener al punto de referencia.

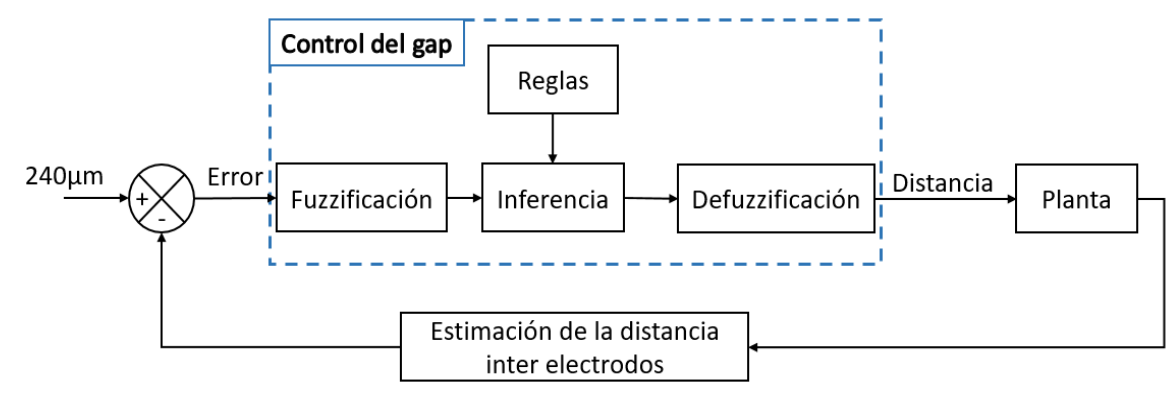

Fig. 3. Modelo SISO del sistema de control para mantener la distancia inter electrodos.

La elección de los conjuntos difusos utilizados se realizó mediante caracterización previa y definir los parámetros de trabajo idóneos del proceso PECMM [16], en la Tabla 3., se muestran las reglas de inferencia generadas. Los conjuntos difusos de entrada utilizados para el control del proceso PECMM, son representados en 3 intervalos, teniendo representaciones de una saturación abierta como $\mu_{L} y$ representaciones triangulares como $\mu_{T}$, como se muestra en la Tabla 1.

Mientras que en la Tabla 2 se presentan los conjuntos difusos de la salida pertenecientes al universo de discurso "Distancia", definidos en tres intervalos contando con formas $\mu_{L} y \mu_{T}$. De acuerdo con Bonifacio Martín del Rio y Alfredo Sanz Molina en [17], el trabajar con conjuntos difusos con formas triangulares; requiere de menor peso computacional y la respuesta a la acción de control se realiza en un menor tiempo que al utilizar conjuntos difusos con formas de tipo campana.

Tabla 1. Conjuntos Difusos del universo de discurso error.

\begin{tabular}{|c|c|c|}
\hline Variable Lingüística & Tipo de Función & Ponderación \\
\hline Negativo & $\mu_{L}$ & $(-60,60)$ \\
\hline Nulo & $\mu_{T}$ & $(-60,0,60)$ \\
\hline Positivo & $\mu_{L}$ & $(-60,60)$ \\
\hline
\end{tabular}


Control de la distancia inter electrodos en un proceso de micro maquinado electroquímico...

Tabla 2. Conjuntos Difusos del universo de discurso Distancia.

\begin{tabular}{|c|c|c|}
\hline Variable Lingüística & Tipo de Función & Ponderación \\
\hline Acércate & $\mu_{L}$ & $(120,480)$ \\
\hline Estable & $\mu_{T}$ & $(120,240,480)$ \\
\hline Aléjate & $\mu_{L}$ & $(120,480)$ \\
\hline
\end{tabular}

Las reglas de inferencia relacionan los conjuntos difusos de entrada y salida para definir el comportamiento del sistema.

Tabla 3. Reglas de inferencia

\begin{tabular}{|c|c|}
\hline Numero de Regla & Reglas de inferencia \\
\hline 1 & Si (Error es negativo) entonces (Distancia es Aléjate) \\
\hline 2 & Si (Error es nulo) entonces (Distancia es Estable) \\
\hline 3 & Si (Error es positivo) entonces (Distancia es Acércate) \\
\hline
\end{tabular}

\section{Resultados}

La validación del sistema de control propuesto se realizó mediante tres pruebas, las cuales se presentan mediante el diagrama de la Fig. 4. La primera se realizó maquinado una pieza de prueba con el eje del movimiento del electrodo paralelo a la superficie de la pieza de trabajo, como se muestra en la Fig. 4(a), la segunda prueba se realizó inclinando la pieza de trabajo un ángulo de $3.5^{\circ}$, Fig. 4(b). Para la tercera prueba se maquinaron canales a lo largo de la superficie de la pieza de trabajo simulando un desnivel sobre la superficie, posteriormente se realizó un maquinado mediante PECMM en dirección perpendicular, Fig. 4(c), al finalizar las pruebas se realizó la medición de la profundidad. Si el sistema de control funciona de manera adecuada, la profundidad del maquinado a lo largo del canal generado mediante el PECMM debe ser de la misma magnitud.

En la Tabla 4, se muestra a manera de resumen la configuración inicial y la profundidad alcanzada al término cada una de las pruebas. La medición de la profundidad de los canales resultantes se realizó al término del maquinado utilizando un medidor de alturas digital marca FLOWLER, modelo 48976.

La configuración que se realiza al principio del proceso PECMM, es establecer el punto de referencia de la corriente de maquinado equivalente a $240 \mu \mathrm{m}$, después el sistema genera una ventana que corresponde al cambio de un paso en sentido positivo y negativo del eje Z, con lo cual se establecen los límites de corriente de maquinado. Dado que el ajuste de altura se realiza mediante motores a pasos, este proceso permite establecer la resolución correspondiente al sistema mecánico.

Posteriormente el control efectúa la acción de mantener constante la distancia inter electrodos y en el momento que detecta que se puede realizar el ajuste de la distancia inter electrodos realiza el reposicionamiento del electrodo, para mantener constante la separación entre la pieza y la herramienta de trabajo a $240 \mu \mathrm{m}$. 


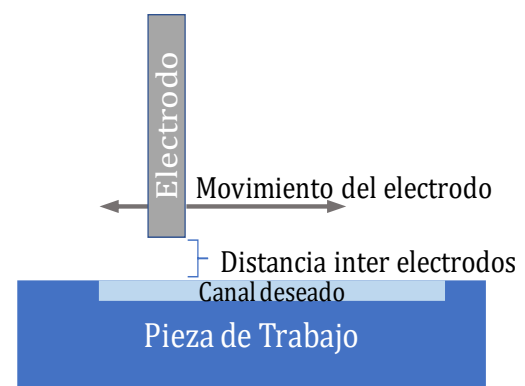

(a)

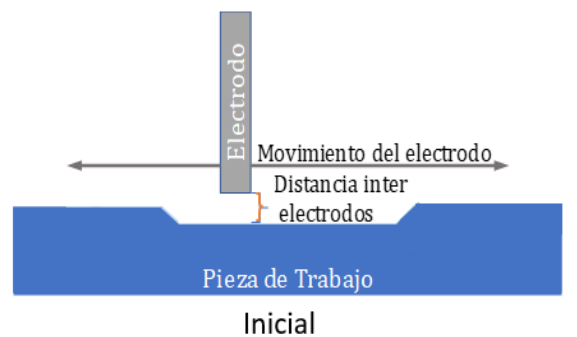

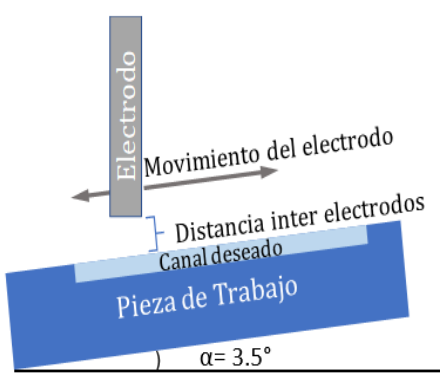

(b)

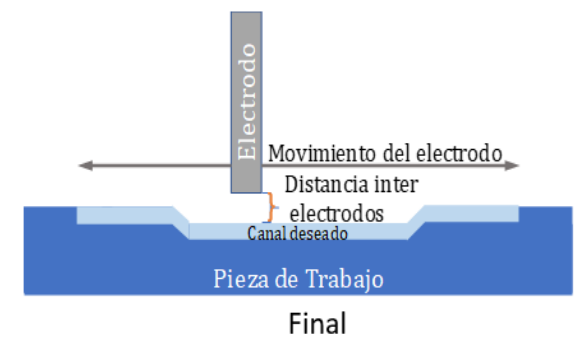

(c)

Fig. 4 . Pruebas propuestas para realizar la validación del sistema de control, (a) Prueba sin perturbaciones, (b) Prueba con la superficie de maquinado inclinada y (c) Prueba sobre una superficie con un canal como perturbación al sistema.

Para generar el canal, el electrodo hace un recorrido constantemente sobre la longitud total del canal, este movimiento se repite hasta obtener el canal deseado, en la Fig. 5(a), se muestra el resultado del maquinado de la prueba 1 y en la Fig. 5(b) el comportamiento de la corriente de maquinado; los resultados de esta prueba son considerados bajo las condiciones más apegadas a la idealidad.

La corriente de maquinado muestra una forma de onda triangular, que corresponde a los ajustes de profundidad que realiza el controlador, de acuerdo a la ecuación 1, la distancia es inversamente proporcional a la corriente RMS de maquinado por lo cual en términos de la distancia, la gráfica representa un aumento del gap dado que se ha removido material de la pieza metálica, hasta un punto que el controlador determina que requiere un reajuste de la distancia, lo que provoca acercar el electrodo a la pieza de trabajo.

En la Fig. 6(a), se muestran los resultados de la prueba 2, al someter el proceso de PECMM a una perturbación que consiste en una inclinación de $3.5^{\circ}$, mientras que la Fig. 6(b) muestra el comportamiento de la corriente de maquinado.

En la forma de onda de la corriente es posible apreciar que la acción de control obliga al proceso a reajustar la posición del electrodo para mantener constante la distancia inter electrodos dentro de la ventana correspondiente a la resolución del sistema mecánico, buscando la estabilidad del proceso. 


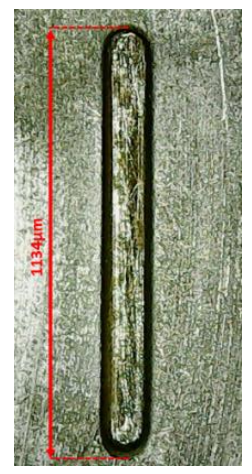

(a)

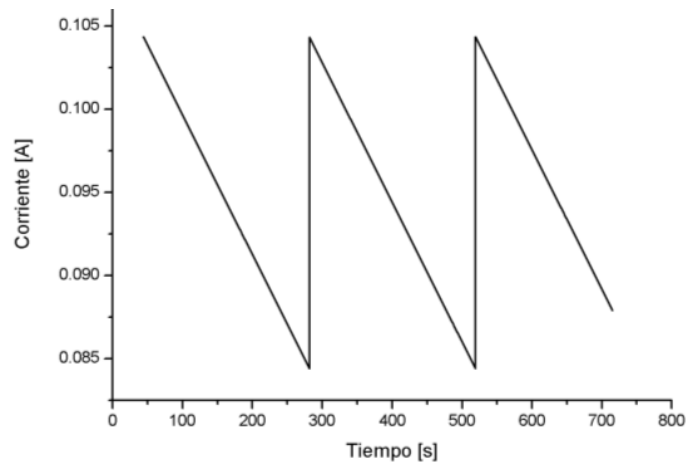

(b)

Fig. 5. (a) Resultados de maquinado sin perturbación y (b) forma de onda de la corriente de maquinado, sin perturbaciones.

Tabla 4. Configuración inicial y mediciones de longitud y profundidad.

\begin{tabular}{|c|c|c|c|c|}
\hline Prueba & $\begin{array}{c}\text { Duración } \\
\text { del } \\
\text { proceso } \\
\text { PECMM } \\
{[\mathrm{min} .]}\end{array}$ & $\begin{array}{c}\text { Longitud } \\
\text { prestablecida } \\
\text { en el sistema } \\
{[\mathrm{mm}]}\end{array}$ & $\begin{array}{c}\text { Longitud } \\
\text { resultante del } \\
\text { proceso } \\
\text { PECMM } \\
{[\mathrm{mm}]}\end{array}$ & $\begin{array}{c}\text { Profundidad } \\
\text { del canal } \\
\text { resultante } \\
{[\mu \mathrm{m}]}\end{array}$ \\
\hline 1 & 15 & 1000 & 1134 & 200 \\
\hline 2 & 15 & 1000 & 1136 & 190 \\
\hline 3 & 30 & 2000 & 2136 & 150 \\
\hline 4 & 25 & 2000 & 2135.6 & 120 \\
\hline 5 & 40 & 2000 & 2132 & 300 \\
\hline 6 & 40 & 2000 & 2131.5 & 300 \\
\hline 7 & 33 & 2000 & 2130 & 250 \\
\hline
\end{tabular}

En la Fig. 7(a), se muestra el resultado del sistema de control, sometido a la prueba 3 , donde la profundidad de la perturbación $1(\mathrm{p} 1)$ es de $330 \mu \mathrm{m}$, mientras que profundidad de la perturbación 2 (p2) es de $270 \mu \mathrm{m}$. En la Fig.7 (b), se muestra la representación de los puntos en donde se realizaron las mediciones de profundidad de cada uno de los canales resultantes. Los resultados de las mediciones de profundidad se muestran en la Tabla 5 y en la Fig. 8, se muestran los puntos de medición al término del maquinado del canal 1 y 2.

El comportamiento de la corriente de maquinado de la prueba 3 se muestra en la Fig. 8 , la forma de la corriente muestra picos abruptos que corresponde a los puntos donde el electrodo se encuentra con el borde de la perturbación, como hay un cambio de distancia entre los electrodos, hay un cambio en la magnitud de la corriente, el 
controlador detecta tales cambios y ajusta la distancia hasta regresar a la magnitud de corriente en que se encontraba antes de presentarse la perturbación.

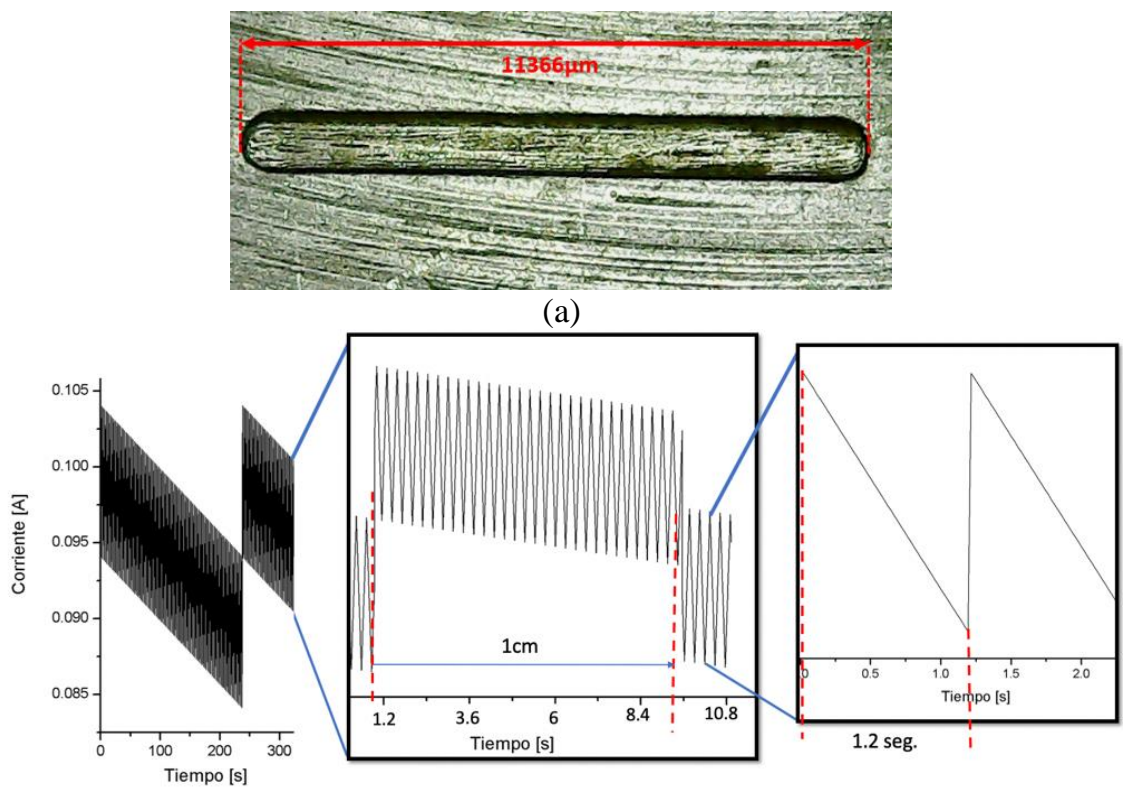

(b)

Fig. 6. (a)Resultados de maquinado con $3.5^{\circ}$ de inclinación y (b) forma de onda de la corriente de maquinado, con una inclinación de $3.5^{\circ}$.

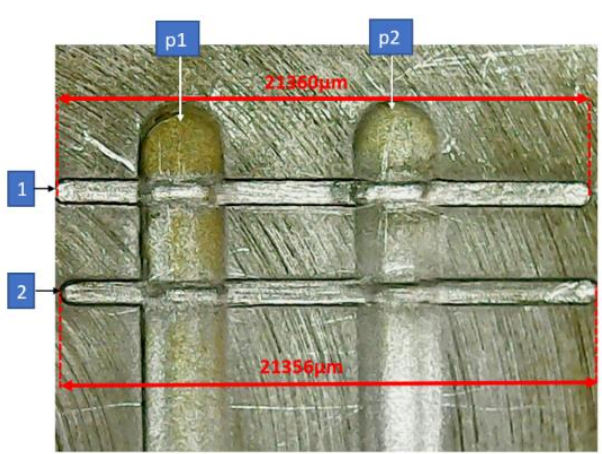

(a)

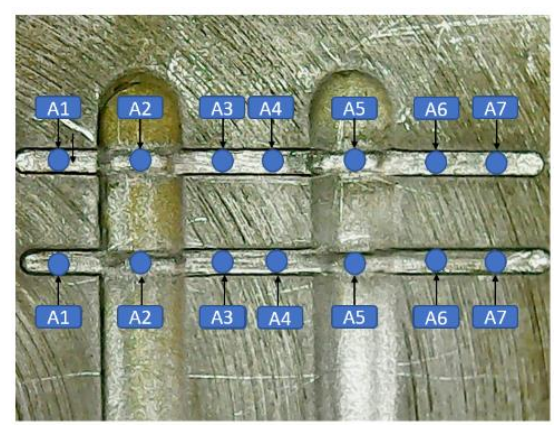

(b)

Fig. 7. (a) Resultado del sistema de control sujeto a perturbaciones, (b) Ubicación de puntos donde se realizaron las mediciones.

Tabla 5. Medición de la profundidad resultante en los canales 1 y 2.

\begin{tabular}{|c|c|c|c|c|c|c|c|c|}
\hline \multirow{2}{*}{ Canal } & \multicolumn{7}{|c|}{ Profundidad $[\mu \mathrm{m}]$} & \multirow{2}{*}{$\begin{array}{c}\text { Media } \\
\end{array}$} \\
\cline { 2 - 9 } & A1 & A2 & A3 & A4 & A5 & A6 & A7 & {$[\mu \mathrm{m}]$} \\
\hline 1 & 150 & 140 & 160 & 150 & 150 & 150 & 150 & $150 \pm 2.9$ \\
\hline 2 & 120 & 110 & 120 & 120 & 120 & 120 & 120 & $118.6 \pm 2.4$ \\
\hline
\end{tabular}


Se presenta solo la forma de onda de la corriente de maquinado para la prueba 3 , dado que las formas de onda son similares.

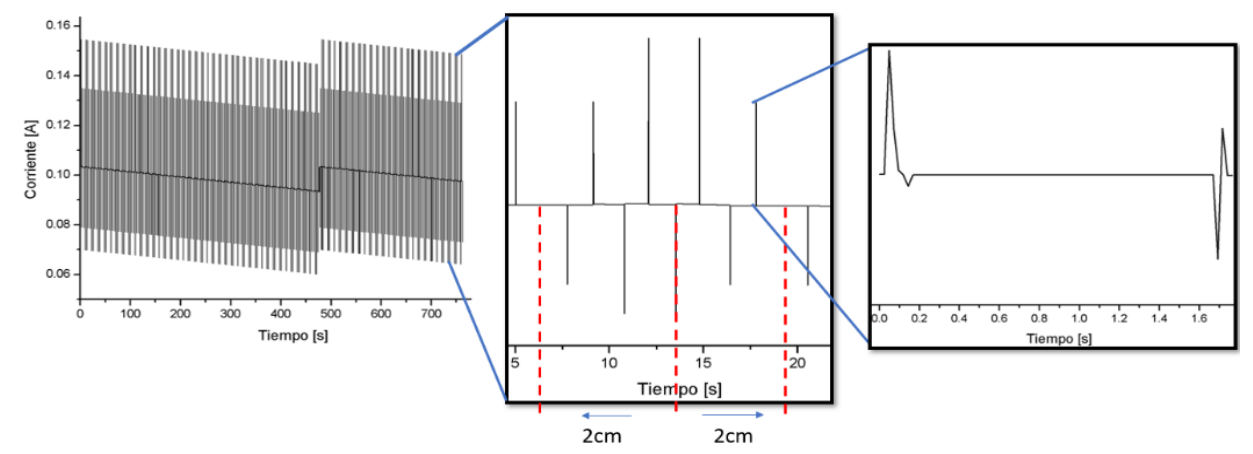

Fig. 8. Comportamiento de la corriente de maquinado del canal 1 (Prueba 3).

En la Fig. 9(a), se muestran los resultados del sistema de control, al ser sometido a una perturbación con un canal lineal vertical creciente, donde la profundidad de la perturbación 3(p3) es un canal con incremento en la profundidad. En la Fig. 9(b), se muestra la representación de los puntos en donde se realizaron las mediciones de profundidad de cada uno de los canales resultantes. Los resultados de las mediciones de profundidad se muestran en la Tabla 6.

La profundidad de la intersección del canal 3 con la perturbación 3, previamente al maquinado fue de $20 \mu \mathrm{m}$, mientras que en la intersección del canal 4 con perturbación 3 la profundidad fue de $160 \mu \mathrm{m}$ y la profundidad del canal 5 con la perturbación 5 de $300 \mu \mathrm{m}$.

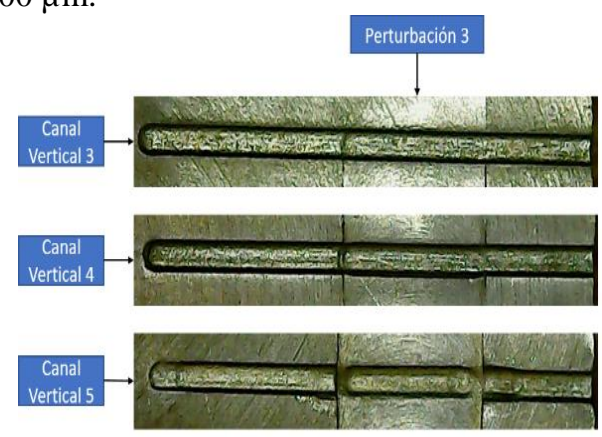

(a)

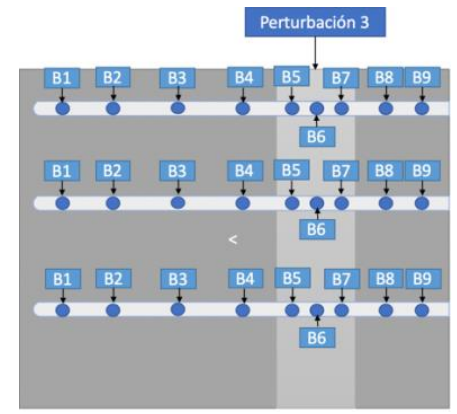

(b)

Fig. 9. (a) Resultados del control del proceso PECMM con perturbación de un canal con profundidad incremental y (b) ubicación de los puntos en donde se realizaron las mediciones.

Tabla 6. Medición de la profundidad resultante en los canales 3, 4 y 5

\begin{tabular}{|c|c|c|c|c|c|c|c|c|c|c|}
\hline \multirow{2}{*}{ Canal } & \multicolumn{8}{|c|}{ Medición $[\mu \mathrm{m}]$} & Media \\
\cline { 2 - 12 } & B1 & B2 & B3 & B4 & B5 & B6 & B7 & B8 & B9 & {$[\mu \mathrm{m}]$} \\
\hline 3 & 310 & 300 & 300 & 300 & 320 & 320 & 320 & 300 & 300 & $301.1 \pm 2.5$ \\
\hline 4 & 310 & 300 & 300 & 300 & 470 & 460 & 460 & 300 & 300 & $302.2 \pm 4.4$ \\
\hline 5 & 260 & 250 & 250 & 250 & 540 & 540 & 540 & 260 & 250 & $248.9 \pm 7.6$ \\
\hline
\end{tabular}




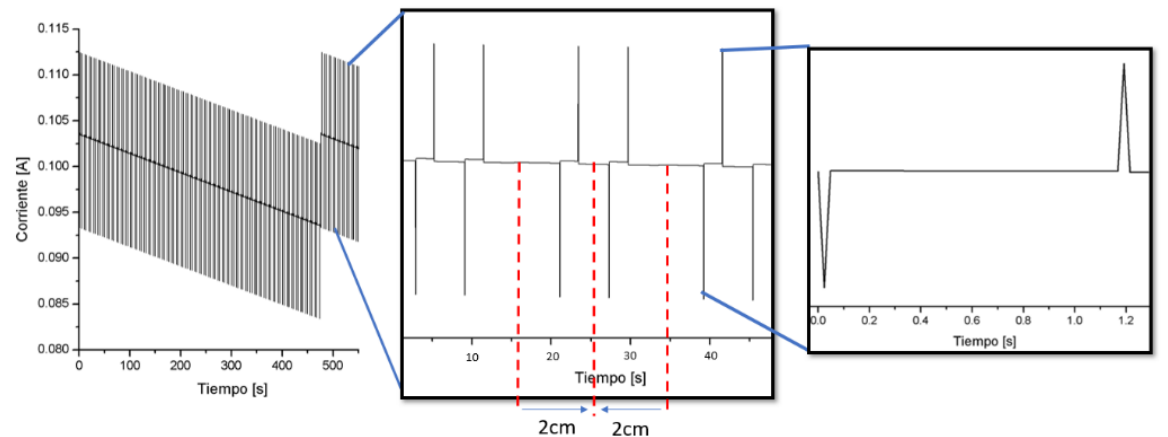

(a)

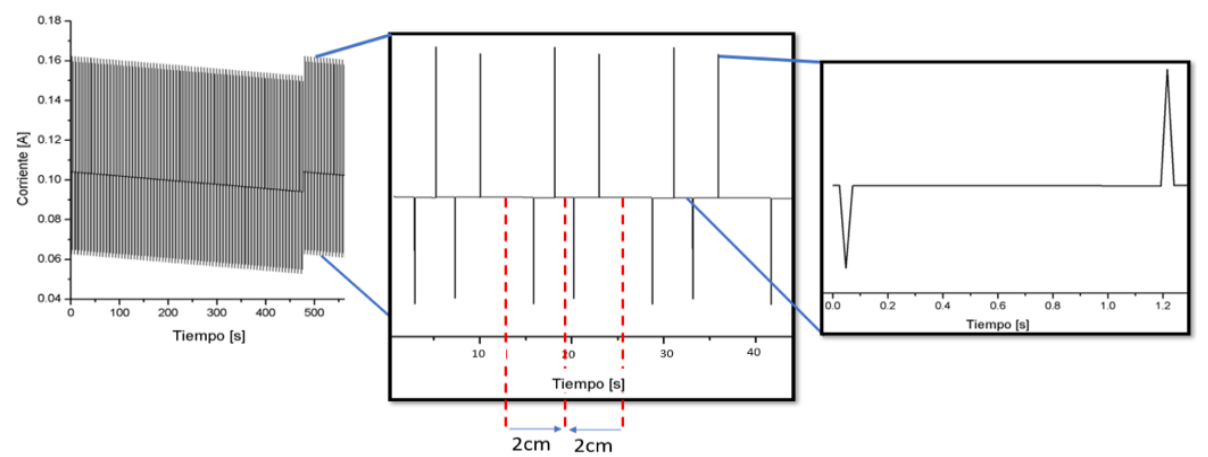

(b)

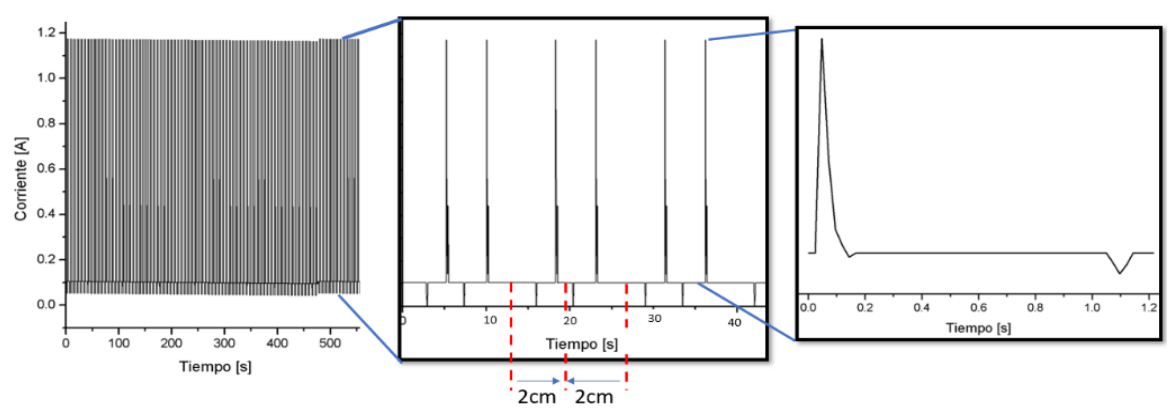

(c)

Fig. 10. Comportamiento de la corriente de maquinado; (a) del canal 3, (b) del canal 4 y (c) del canal 5.

La forma de onda de la corriente de maquinado es similar a las pruebas realizadas en la prueba 3 y 4, es decir, al recorrer el electrodo la longitud del canal que se desea maquinar, en el trayecto encuentra perturbaciones de diferente magnitud (el canal 3 con menor magnitud, mientras que el canal 5 con mayor magnitud).

Dichas perturbaciones se ven reflejadas en la forma de corriente y el distanciamiento entre cada pico de corriente coincide con la distancia recorrida por el electrodo. La acción de control se ve reflejada cuando el electrodo cae dentro del canal de 
perturbación, el gap aumenta drásticamente y la corriente disminuye, el controlador ajusta la distancia hasta que la corriente regresa a la condición de estabilidad (magnitud de corriente de maquinado).

En caso opuesto, si el electrodo sale del canal de perturbación, el gap disminuye abruptamente y corriente aumenta; el controlador ajusta la distancia alejándose de la pieza de trabajo hasta que la corriente regresa a la condición de estabilidad.

En la Fig. 10, se muestra el comportamiento de la corriente de maquinado de los canales 3, 4 y 5. La diferencia entre las figuras es la magnitud de los picos de corriente, y es debido a las diferentes profundidades que se presentan en las perturbaciones. Para el canal 3, el pico de corriente es de $112 \mathrm{~mA}$; para el canal 4 es de $164 \mathrm{~mA}$ y para el canal 5 es de $1.185 \mathrm{~A}$.

\section{Conclusiones}

El presente trabajo se muestra el desarrollo de un sistema de control difuso que mantiene constante la distancia entre electrodos. De acuerdo a la literatura y a experimentación previa, el PECMM es un proceso de maquinado complejo, en el cual intervienen fenómenos, físicos, químicos y eléctricos, donde una característica que se requiere cumplir es mantener constante la distancia entre los electrodos para establecer una remoción homogénea de material. Al momento no existe herramienta que permita realizar la medición de dicha distancia, una alternativa es estimarla mediante el monitoreo de la corriente de maquinado.

El controlar el proceso PECMM a través de sistemas clásicos requiere de conocer el modelo matemático de la planta, lo cual resulta complejo de obtener, ya que involucra diversas y diferentes variables. Una alternativa viable presentada en este trabajo es mediante un control inteligente, de forma específica por medio de lógica difusa. El diseño y aplicación del regulador difuso tipo SISO con inferencias tipo Mamdani permite mantener dentro de un intervalo adecuado de operación el proceso PECMM, los resultados permiten validarlo, inclusive con las limitantes mecánicas con que está construido el sistema pues el ajuste se realiza con motores a pasos. Se realizaron tres diferentes pruebas para validar el controlador, la primera es ajustando la pieza para considerar los parámetros idóneos.

En esta prueba se visualiza el ajuste continuo de la distancia conforme va removiendo material. La segunda prueba se realiza al inclinar la pieza de trabajo, donde el controlador ajusta continuamente la distancia, manteniendo el gap lo más cercano a una constante.

La tercera prueba fue generando irregularidades en la pieza de trabajo consideradas como perturbaciones, en la forma de onda de la corriente de maquinado se puede visualizar cuando el controlador ajusta la distancia hasta regresar a un valor de corriente previa a la detección de la perturbación.

El resultado en cualquier de las pruebas es un canal uniforme, con un error máximo de $7.6 \mu \mathrm{m}$. Como trabajo a futuro se debe mejorar la respuesta mecánica del proceso; además de realizar una comparativa de método aquí presentado con otros métodos. 


\section{Referencias}

1. Grover P, M.: Fundamentos de Manufactura Moderna. 4th edn. Mc Graw Hill, México (2010)

2. Kalpakjian, S., Schmid, S.R.: Manufactura, Ingeniería y Tecnología, 2nd edn. Prentice Hall, México (2005)

3. Hassan, A., Gawad, E.-H.: Advanced Machining Processes, 5th edn. McGraw-Hill, Alexandia (2005)

4. Katz, Z., Tibbles J.C.: Analysis of micro-scale ECM Proccess. The International Journal of Advance Manufacturing Technology 35(154), 923-928 (2012)

5. Sengottuvel, P., Satishkumar, S., Dinakaran, D.: Optimization of Multiple Characteristics of EDM Parameters Based on Desirability Approach and Fuzzy Modeling. Procedia Engineering 64, 1069-1078 (2013)

6. Shabgard, M., Badamchizadeh, M.G., Ranjbary, K.: Fuzzy approach to select machining parameters in electrical discharge machining (EDM) and ultrasonic-assisted EDM processes. Journal of Manufacturing Systems 32(1), 32-39 (2013)

7. Mohd, A., Sarkheyli, A., Mohd Zain, A., Habibollah, H.: Fuzzy logic for modeling machining process: a review. Artificial Intelligence Review 43(3), 345-349 (2013)

8. Katz, Z., Tibbles J.C.: Analysis of micro-scale ECM Proccess. The International Journal of Advance Manufacturing Technology 35(154), 923-928 (2012)

9. Klocke, F.: Results of Surface Integrity and Fatigue Study of PECM and PEO processed $\gamma$ TiAl for Turbine Applications. Procedia CIRP19 vol. 3, pp. 131-136 (2016)

10. Lilong Baoji, M., Cheng, P., Yun, K.: Effect of Power Signal Waveform on Shape Accuracy in Electrochemical Drilling. Materials Science and Engineering 392, pp. 1-8 (2018)

11. Kohli, A., Wadhwa, A., Virmani, T., Jain, U.: Optimization of Material Removal Rate in Electrical Discharge Machining Using Fuzzy Logic. World Academy of Science, Engineering and Technology International Journal of Mechanical and Mechatronics Engineering 12, pp. 2839-2844 (2012)

12. Hung Lea, V., Khanh Tran, D.: Extending fuzzy logics with many hedges. Fuzzy Sets and Systems 1(1), 45-52 (2018)

13. Keasberry, J.V., Labib, W.A., Atkinson, J., Frost W.H.: A Fuzzy Logic Control Approach to Electrochemical Machining (ECM). In: Proceedings of the 34th International, Formerly the International Machine Tool Design and Research Conferences 34, pp. 153-160 (2008)

14. Sadegh, A.M.: Controlling Electrochemical Machining by Using a Fuzzy Logic Approach. Journal of Modern Processes in Manufacturing and Production 6(2), 61-71 (2017)

15. Martínez Alvarado, R., Granda Gutiérrez, E.E., Zuñiga, L.M., Guerrero, R.: Pulsed Power Supply for Electrochemical Machining. In: 2015 IEEE International Autumn Meeting on Power, Electronics and Computing (ROPEC) 3(15), 1-6 (2015)

16. Gallegos Miranda, I., Pérez Martínez, J.A., Granda Gutiérrez, E.E., Flores Fuentes, A.A.: Caracterización de Proceso de Maquinado Electroquímico por Pulsos (PECM). En: Coloquio de Investigación Multidisciplinaria 6 (1), 414-421 (2018)

17. Martín del Río, B., Sanz Molina, A.: Redes Neuronales y Sistemas Borrosos. 3rd edn. Alfaomega, México (2007) 